\title{
The Prevalence and Management of Pauci-Immune Glomerulonephritis and Vasculitis in Western Countries
}

\author{
Sophia Lionaki ${ }^{a, b} \quad J o h n N$. Boletis ${ }^{a, b}$ \\ ${ }^{a}$ Department of Nephrology and ${ }^{b}$ Transplantation Unit, Laiko Hospital, Athens, Greece
}

\section{Key Words}

Epidemiology · Glomerulonephritis · Management ·

Pauci-immune $\cdot$ Vasculitis

\begin{abstract}
Background: Pauci-immune glomerulonephritis is the most common cause of aggressive glomerulonephritis and occurs as a renal-limited disease or as a component of systemic necrotizing small-vessel vasculitis. It is characterized by paucity of staining for immunoglobulins, by immunofluorescence along with fibrinoid necrosis and crescent formation by light microscopy, while the vast majority of patients have antineutrophil cytoplasmic antibodies (ANCA) in their circulation, which also participate in the pathogenesis of the disease. Summary: Pauci-immune glomerulonephritis often manifests with rapidly deteriorating kidney function, which may be accompanied by distinctive clinical features of systemic necrotizing small-vessel vasculitis of one the following clinical phenotypes: microscopic polyangiitis, granulomatosis with polyangiitis or eosinophilic granulomatosis with polyangiitis. These are associated with a wide spectrum of vasculitic manifestations in different organ systems at clinical presentation and during the course of the disease. ANCA specificity is associated with distinct clinical syndromes and different prognostic profiles among patients. The key element of the management of patients with pauci-immune glomerulonephritis, with or without systemic vasculitis, is
\end{abstract}

the clinical acumen, which results in timely diagnosis. Speed in diagnosis is crucial for the quick institution of immunosuppressive therapy aimed at removing circulating autoantibodies and quelling the inflammatory process. Key Messages: The introduction of ANCA testing in routine clinical practice has increased the ability of disease suspicion and recognition, resulting in earlier establishment of diagnosis by seeking a tissue confirmation of pauci-immune vasculitis. ANCA specificity is associated with distinct clinical syndromes and different prognostic profiles among patients. The management of patients with ANCA glomerulonephritis and/or vasculitis includes two major elements: prompt diagnosis and institution of immunosuppressive therapy to avoid irreversible kidney damage or death, and consideration of the predictors, which are associated with relapsing disease for planning of therapy in the long term. Facts from East and West: Treatment options for ANCA-associated vasculitis are shared between the East and West, with corticosteroid combined with cyclophosphamide being the standard regimen for inductive therapy and switching to azathioprine after remission. The major cause of death in treated patients is infection related to immunosuppressive therapy within the first year after diagnosis, and this rate might be higher in China than in Western countries. Western studies demonstrated

For the prevalence and management of anti-neutrophil cytoplasmic antibody-associated vasculitis in China, see Li et al., Kidney Dis 2015; 1:216-223.

\section{KARGER 125\%}

(c) 2015 S. Karger AG, Base

$2296-9381 / 15 / 0014-0224 \$ 39.50 / 0$
Sophia Lionaki, $\mathrm{MD}, \mathrm{PhD}$

Ag Thoma 17

GR-11527 Athens (Greece)

E-Mail sofia.lionaki@gmail.com 
the efficacy and safety of rituximab for induction of remission in cases with relatively mild disease and maintenance therapy, but this agent is rarely used in China.

(C) 2015 S. Karger AG, Basel

\section{Introduction}

Pauci-immune glomerulonephritis, which is characterized by the paucity of staining for immunoglobulins, is the most common cause of crescentic glomerulonephritis (i.e. with $50 \%$ or more glomeruli being involved with crescents) and may occur as a renal-limited disease or as a component of systemic necrotizing small-vessel vasculitis [1]. The systemic vasculitides that may be accompanied by pauci-immune crescentic glomerulonephritis include microscopic polyangiitis (MPA), granulomatosis with polyangiitis (GPA) and eosinophilic GPA (EGPA) [2]. The vast majority (85-90\%) of patients with active untreated pauci-immune crescentic glomerulonephritis and vasculitis are positive for anti-neutrophil cytoplasmic antibodies (ANCA) [1], and therefore, it is called ANCAassociated vasculitis (AAV). Distinct clinical syndromes and disease profiles have been associated with each ANCA type.

This review will focus on the prevalence of pauci-immune glomerulonephritis and vasculitides in Western countries and the current therapeutic options for these diseases. Recent advances in the understanding of correlations between laboratory, serological and clinical prognostic parameters, as well as related therapies will be also discussed.

\section{Epidemiology}

Pauci-immune crescentic glomerulonephritis is the most common cause of aggressive glomerulonephritis [3-5] and it is more frequent in older patients, with equal distribution between genders [5-7]. The incidence of pauci-immune glomerulonephritis, with or without small-vessel vasculitis, in the United States has been found to be 3.1 cases/million/year, with rates being significantly higher for Caucasians, males and individuals older than 65 years, while 95\% of cases were ANCA-positive at the time of biopsy $[6,8]$. In the European population, the incidence is reported to be 1-2 cases in 100,000 $[7,9]$, with an increasing trend in recent years up to 2000 [10], which may be a consequence of an actual increase in disease frequency, increased awareness among physi-

Pauci-Immune Glomerulonephritis and Vasculitis in Western Countries cians, and better ability of disease recognition through the introduction of ANCA testing. However, since the incidence has been stable since the early 2000s, it is most likely that increased physician awareness following the introduction of ANCA testing in routine clinical practice is the most likely reason [11]. Moreover, the incidence of GPA is higher than that of MPA in Northern Europe, while MPA is predominant among cases of AAV in Southern Europe [11-13].

The prevalence of AAV has been estimated to be $46-$ 184 cases/million [11], with the rate having increased during the last two decades. The main explanation for this phenomenon is probably the fact that patient survival has improved significantly during this period as treatment options are more effective and physicians have a higher level of suspicion of the disease. Genetic factors definitively play a role, as has been shown from studies which underline the fact that AAV is relatively rare in non-Caucasian populations [11]. A study in a multi-ethnic cohort of patients from the University of North Carolina at Chapel Hill (USA) showed that GPA is quite uncommon in African Americans [14]. Interestingly, the investigators showed that the HLA-DRB1*15 allele is a risk factor for PR3-ANCA disease in African Americans [14]. It is probable that there is a global variation of the HLA$\mathrm{DRB}^{*} 15$ allele, which is also reflected in the variation of clinical phenotypes of the disease across different geographical areas. Another study, which directly compared two ethnically different populations - white Caucasians from the UK and Japanese - showed that although the incidence rate overall was comparable, GPA and PR3ANCA vasculitis were much less common in Japan than in Europe [11].

\section{Pathology}

\section{Renal Pathology}

The hallmark histological lesions of acute pauci-immune glomerulonephritis are crescents and fibrinoid necrosis (fig. 1) occurring with the same frequency, irrespective of the presence or absence of associated vasculitis [1]. The severity of acute lesions ranges from focal segmental fibrinoid necrosis affecting $<10 \%$ of glomeruli to severe diffuse necrotizing and crescentic glomerulonephritis, which may injure all glomeruli [1] (fig. 2). The extent of crescent formation does not differ between patients with PR3-ANCA or MPO-ANCA [4]. Breaks in Bowman's capsule are common [15]. Periglomerular granulomatous inflammation may occur but is not spe- 


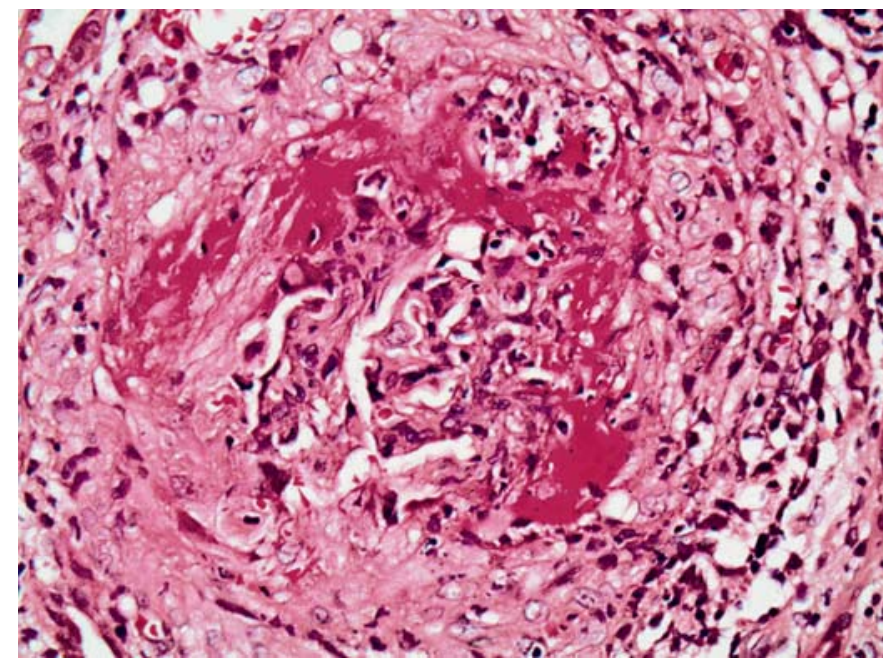

Fig. 1. Glomerulus from a patient with PR3-ANCA pauci-immune crescentic glomerulonephritis showing remarkable fibrinoid necrosis surrounded by crescent formation. H\&E, $\times 400$.

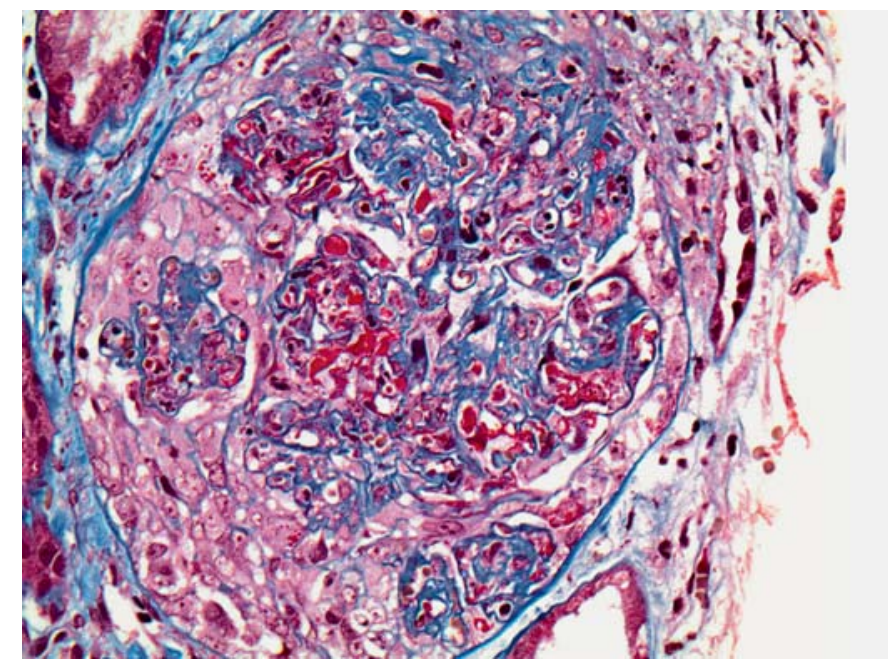

Fig. 2. Glomerulus from a patient with MPO-ANCA pauci-immune crescentic glomerulonephritis stained with Masson trichrome showing well-formed cellular crescent and segmental fibrinoid necrosis that stained red with the Masson trichrome stain.

staining for immunoglobulin (i.e. $<2$ on a $0-4$ scale) [1]. Therefore, in the absence of renal involvement, the diagnosis of ANCA vasculitis should be confirmed by tissue biopsy, at any site of active disease in any organ. However, immunosuppressive treatment should be started empirically if the clinical suspicion of ANCA vasculitis is high and a tissue diagnosis cannot be obtained in a timely manner [6]. Among patients with MPA the typical histological lesion in the lungs is pulmonary capillaritis, while among patients with GPA, granulomatous inflammation may be seen. Necrotizing granulomatous inflammation most often affects the upper and lower respiratory tract but may occur anywhere, such as in the dermis and subcutaneous tissue. Granulomas are characterized histologically by an irregular central zone of necrosis that may have an amphophilic or bluish hue because of finely dispersed nuclear debris [1]. Epithelioid macrophages may be numerous although they do not have the compact arrangement seen in other occasions such as sarcoidosis [1]. More chronic lesions have extensive fibroblastic proliferation and ultimately evolve into dense fibrotic scars. Nevertheless, for any specimen with necrotizing granulomatous inflammation, major non-vasculitic differential diagnostic considerations, including mycobacterial and fungal infections, should be made and ruled out [1]. Thus, several sites of extra-renal involvement may be used to obtain a tissue biopsy, such as the skin, the lung or the nose. A lung biopsy most often requires an open or tho- 
racoscopic lung procedure. In a small proportion of patients, sufficient tissue for diagnosis can be obtained by transbronchial biopsy of the lung; however, the absence of granulomatous vasculitis on transbronchial specimens should not be considered adequate evidence to exclude the diagnosis of GPA [17]. A nasal biopsy, on the other hand, although relatively easy and non-invasive, is limited by the high rate of false-negative results, since the amount of tissue that can be removed is small. A positive lung biopsy establishes the diagnosis in such cases and from one view precludes the need for a kidney biopsy in many cases; however, a renal biopsy is still indicated in patients who are initially diagnosed by lung biopsy, especially if they experience a rapidly progressive course of renal dysfunction, in order to assess long-term prognosis and plan immunosuppressive therapy accordingly.

\section{The Role of ANCA in Disease Recognition and Pathogenesis}

ANCA are antibodies specific for proteins in the cytoplasmic granules of neutrophils and the lysosomes of monocytes. They were first reported by Davies et al. [18], while in 1988, it was demonstrated that the vast majority of patients with pauci-immune crescentic glomerulonephritis have ANCA in their circulation, including patients with or without systemic vasculitis [19]. Two major specificities for ANCA are distinguished on the basis of cytoplasmic (cANCA) or perinuclear (pANCA) ANCA staining by indirect immunofluorescence microscopy and by enzyme immunoassay demonstrating anti-myeloperoxidase (MPO-ANCA) and anti-proteinase 3 (PR3ANCA). Approximately $90 \%$ of cytoplasmic ANCA are PR3-ANCA, and approximately $90 \%$ of perinuclear ANCA are MPO-ANCA. Patients with GPA usually have cANCA (PR3-ANCA), patients with MPA have slightly more pANCA (MPO-ANCA), and patients with EGPA and renal-limited AAV have predominantly pANCA (MPO-ANCA) [1]. More importantly, ANCA specificity was shown to be strongly associated with organ involvement and distinct clinical pictures of pauci-immune vasculitis. Specifically, patients with kidney-limited disease or any form of vasculitis without radiological or histological proof of granulomatous inflammation were more likely to have MPO-ANCA, and those with evidence for necrotizing granulomatous inflammation were most likely to have PR3-ANCA. In a study of 523 patients the majority of those with kidney-limited disease had MPOANCA (81\%), while almost all patients with bone de-

Pauci-Immune Glomerulonephritis and

Vasculitis in Western Countries struction or saddle nose deformity had PR3-ANCA (94\%) [20]. The association between PR3-ANCA or MPOANCA and the anatomic site of vasculitic involvement and/or the presence of granulomatous inflammation was strong and impressive. When vasculitis expanded from the kidneys to involve the gastrointestinal or the respiratory tract, MPO-ANCA was less frequent and PR3ANCA was increased. Moreover, among 52 patients with histological proof of granulomatous inflammation at any site in our cohort, 79\% had PR3-ANCA and 21\% had MPO-ANCA. Therefore, the principle that diseases associated with MPO-ANCA and PR3-ANCA are clinically distinct is proven essential to the categorization of ANCA small-vessel vasculitis based on antibody specificity [20].

In accordance with this observation, a genome-wide association study confirmed that the pathogenesis of AAV has a genetic component, showing genetic distinctions between GPA and MPA, which are associated with ANCA specificity, indicating that the response against the autoantigen PR3 is a central pathogenic feature of PR3 AAV [21].

A certain pathogenic role of ANCA has been demonstrated by a large number of in vitro studies, which reveal that both PR3-ANCA and MPO-ANCA IgG activate neutrophils that then release mediators of acute inflammation [22-25]. Neutrophils activated by ANCA IgG can kill cultured endothelial cells under certain conditions. It has also been shown that activation of neutrophils by ANCA causes integrin and cytokine receptor mediated adherence to cultured endothelial cells and transmigration across the endothelial layer [26], as well as a conformational change in $\beta$ integrins which enhances ligand binding. Unregulated adhesion molecules in glomerular lesions of renal biopsy specimens from patients with AAV support their role in the interaction of ANCA-activated neutrophils with vessels [27].

However, the most compelling evidence that ANCA are pathogenic comes from in vivo studies and specifically from a mouse model in which passive transfer of anti-MPO IgG (MPO-ANCA) or anti-MPO lymphocytes led to induction of glomerulonephritis. Xiao et al. [28] developed a model in which intravenous administration of anti-MPO IgG into either immune-competent mice or Rag2-/- mice that have no functioning T or B cells causes pauci-immune crescentic glomerulonephritis and smallvessel vasculitis, remarkably similar to human disease. Within 6 days of the intravenous injection, all mice develop glomerular lesions while some develop systemic vasculitis, including leukocytoclastic angiitis, necrotizing arteritis, pulmonary capillaritis and necrotizing granulo- 
matosis inflammation [28-31]. Similarly, severe crescentic glomerulonephritis and systemic vasculitis can be induced by passive transfer of splenocytes from MPO-/mice that have been immunized with murine MPO. The renal injury in this model is exacerbated by stimulation with lipopolysaccharide [30] and appears to be dependent on an intact alternative complement pathway and the presence of neutrophils [32]. Another model produced focal segmental pauci-immune glomerulonephritis and focal pulmonary capillaritis in rats by immunization with human MPO, which induced anti-MPO antibodies that cross-react with human and rat MPO [33].

Interestingly, there are patients with clear clinical and histopathological evidence of small-vessel vasculitis and/ or pauci-immune necrotizing and crescentic glomerulonephritis with repetitively negative serology for ANCA. This issue is important basically because the negative serology often causes delays in establishing the diagnosis and initiating appropriate immunosuppressive treatment. To address this issue, a multicenter study recently reported the development of a novel assay to identify the specific target epitopes for ANCA [34]. This methodology led to the discovery of MPO-ANCA in patients with ANCA-negative disease that reacted against a sole linear sequence. Autoantibodies against this epitope had pathogenic properties, as demonstrated by their capacity to activate neutrophils in vitro and to induce nephritis in mice. The confounder for the serological detection of these autoantibodies was the presence of a fragment of ceruloplasmin in serum, which was eliminated in purified IgG, allowing detection. These findings implicate immunodominant epitopes in the pathology of AAV and suggest that autoantibody diversity may be common to other autoimmune diseases [34].

\section{Clinical Presentation of Renal Disease}

Patients with pauci-immune glomerulonephritis may present with different clinical pictures starting from asymptomatic hematuria to more severe forms of glomerulonephritis such as rapidly progressive glomerulonephritis. Typically, they have active urine sediment and acutely elevated serum creatinine with microscopic urine analysis characteristically demonstrating dysmorphic erythrocyturia, with or without red cell casts and proteinuria. In a prospective study of 70 patients with pauci-immune glomerulonephritis followed for 24 months, hematuria was a common finding as was proteinuria, with a mean protein excretion of $2.5 \mathrm{~g} / 24 \mathrm{~h}$ and a mean entry serum creatinine of $5.5 \pm 4.1 \mathrm{mg} / \mathrm{dl}$. Of these patients, $24.3 \%$ required dialysis at onset or during follow-up [35]. General symptoms including low-grade fever, fatigue, weight loss, myalgias and arthralgias often precede the actual presentation of the disease $[35,36]$. The vast majority of patients (94\%) reported a prodromal 'flu-like' illness preceding the overt vasculitic syndrome [35]. Approximately $75 \%$ of patients with pauci-immune glomerulonephritis have manifestations of systemic small-vessel vasculitis at presentation [37] or develop them over time [38]. Clinical or pathological evidence of renal disease is seen in approximately $90 \%$ of patients with MPA, $80 \%$ of patients with GPA and $45 \%$ of patients with EGPA [39$45]$. The distribution of clinical phenotypes of AAV in a cohort of 350 ANCA-positive patients with pauci-immune crescentic glomerulonephritis was $58 \%$ for MPA, $25 \%$ for renal limited disease and 17\% for GPA [8]. MPA, GPA and EGPA share certain clinical features, but each also has distinctive characteristics [39-43, 46, 47].

\section{Spectrum of Extra-Renal Vasculitic Manifestations}

Upper respiratory tract disease, in different forms and degrees of severity, is present in $90 \%$ of patients with GPA $[36,41]$. It includes nasal crusting and obstruction, bloody nasal discharge or epistaxis-related nasal mucosa ulceration, sinus pain with associated drainage and otitis media; hearing loss may be seen. Involvement of the vessels supplying the cartilage may lead to septal perforation or destructive bone disease with or without granulomas invading the anatomy in patients with GPA. Saddle nose deformation due to collapse of the nasal structure and facial paralysis due to facial nerve entrapment may occur, but the inflammation of the trachea, often in the subglottic region, is the most dangerous condition, resulting in airway stenosis. However, subglottic stenosis and destruction in the sinonasal anatomy represent exclusive manifestations of the GPA phenotype.

Pulmonary involvement, on the other hand, may present as necrotizing granulomatous inflammation or alveolar capillaritis and is typically demonstrated on radiographic studies as nodular opacities or alveolar infiltrates. Pulmonary nodules may cavitate, thus making disease management highly problematic in the setting of superimposed infections. Capillaritis, arteritis and granulomatous inflammation may cause hemoptysis or massive pulmonary hemorrhage, life-threatening conditions which require immediate induction therapy. In a cohort of 70 patients with biopsy-proven pauci-immune glomerulo- 
nephritis, $53 \%$ were found to have pulmonary disease manifested as hemoptysis or massive pulmonary hemorrhage that rapidly became fatal in half of the cases [35].

Cutaneous involvement with palpable purpura and nodules occurs in as many as $25 \%$ of patients. Other skin lesions include erythematous macular lesions, papules, infarcts and necrotic ulcers. Vasculitic lesions in the mucous membranes appear as aphthous stomatitis or oral ulceration. Ophthalmologic manifestations include conjunctivitis, episcleritis, blepharitis, keratitis, acute visual loss or orbital mass. Approximately $30 \%$ of patients report symptoms of abdominal pain, gastritis, ischemic colitis or pancreatitis due to involvement of the vessels in the abdominal organs. Occasional infarction of the bowel with viscus perforation and polymicrobial sepsis may result in life-threatening phenomena. Cranial nerve palsy, sensory peripheral neuropathy or mononeuritis multiplex may be seen. Peripheral neuropathy caused by vasculitis in epineural arterioles and arteries occurs in approximately $30 \%$ of patients. Vessels in the central nervous system can also be affected, leading to sudden onset of seizures, cerebrovascular events and cognitive disorders. Deep vein thrombosis may also occur more frequently among patients with AAV in comparison with the general population, or other autoimmune disorders [48]. Interestingly, anti-plasminogen autoantibodies have been identified in patients with PR3-ANCA glomerulonephritis as a result of utilization of a peptide coded by the anti-sense RNA of the PRTN3 gene [49].

EGPA represents a somewhat different disease manifested by asthma, eosinophilia and granulomatous inflammation in the lung. ANCA are positive in $70 \%$ of patients, most commonly MPO-ANCA [40], and eosinophilia $>10 \%$ in the peripheral blood is found. Coronary arteritis and myocarditis are the main causes of morbidity and mortality, accounting for $50 \%$ of deaths. Renal disease is much less frequent and less severe in this disease category, in contrast with neuropathy and cardiac disease, which are more common. However, recent advances in EGPA suggest that the majority of patients who are ANCA-positive also have glomerulonephritis, while those lacking ANCA are more likely to have cardiac disease [50].

\section{Response to Immunosuppressive Therapy and Long-Term Outcomes}

Immunosuppressive therapy in AAV aims at induction of remission, defined as stabilization or improvement of kidney function, resolution of hematuria and all other organ-specific vasculitic manifestations [51]. Inadequate or no response to therapy is considered treatment resistance, defined as a progressive decline in kidney function, with persistent active urine sediment, or persistence, or new appearance of any extra-renal manifestations of vasculitis, despite immunosuppressive treatment. There are also patients who initially responded to therapy in a manner that let them escape life-threatening or advanced organ damage, yet never achieved complete obliteration of the pathogenic process, maintaining a low grade of persistent 'grumbling disease'. Patients achieving remission, either complete (off immunosuppressive therapy) or on therapy, may or may not experience one or more relapses afterwards. These are manifested as vasculitic signs or symptoms in any organ system, although relapses tend to affect the same organ systems as on initial presentation, with new organ involvement reported only in $23 \%$ of patients [51].

\section{Inductive Therapy}

The gold standard of treatment in AAV is the combination of corticosteroids with the cytotoxic agent cyclophosphamide $[6,41,47,52]$, given as intravenous pulses of methylprednisolone $(7 \mathrm{mg} / \mathrm{kg}$ body weight for 3 consecutive days), followed by oral prednisone $(1 \mathrm{mg} / \mathrm{kg}$ body weight for the first 4 weeks), reduced in a gradual and personalized manner over the next 3-5 months. Cyclophosphamide is given either monthly, intravenously starting at a dose of $0.5 \mathrm{~g} / \mathrm{m}^{2}$ of body surface area, subsequently increased up to $1 \mathrm{~g} / \mathrm{m}^{2}$ of body surface area, or orally at an initial dose of $2 \mathrm{mg} / \mathrm{kg}$ of body weight/day, always adjusted on the basis of the patient's leukocyte count and the current glomerular filtration rate. The duration of therapy with cyclophosphamide is usually 6-12 months, depending on the patient's initial response [35, 51]. Both oral and intravenous administrations of cyclophosphamide have been proven equally potent inductors of remission $[35,53]$ in AAV, but its cumulative dose is significantly lower in parenteral administration. A multivariate analysis showed superior remission rates for the intravenous regimen without significantly higher relapse rates [53]. Yet, another study with a retrospective design [54] showed that treatment with pulse cyclophosphamide is associated with a higher relapse risk than the oral regimen, but this was not associated with increased frequency of end-stage renal disease (ESRD), mortality or longterm morbidity [54]. 
Rituximab, a chimeric monoclonal antibody directed against the CD20 antigen of B lymphocytes, has also been used in the inductive treatment of AAV, either in combination with steroids or with cyclophosphamide and steroids $[55,56]$. Jones et al. [55] compared rituximab with cyclophosphamide, as induction therapy, in patients with newly diagnosed AAV and renal involvement, to a standard glucocorticoid regimen plus either rituximab with two intravenous cyclophosphamide pulses, or intravenous cyclophosphamide for 3-6 months followed by azathioprine. The rituximab-based regimen was not inferior to standard intravenous cyclophosphamide, as sustained remission rates were high in both groups, but also the rituximab-based regimen was not associated with reductions in early severe adverse events. The second study, which enrolled 197 ANCA-positive patients with either GPA or MPA and relatively mild disease compared treatment with rituximab to treatment with oral cyclophosphamide for remission induction. The rituximab-based regimen was shown to be more efficacious than the cyclophosphamide-based regimen for inducing remission in patients with relapsing disease [56].

There are two clear indications which justify addition of plasma exchange in the inductive phase of treatment in AAV: pulmonary hemorrhage and severe renal dysfunction at clinical presentation (serum creatinine $>500$ $\mu \mathrm{mol} / \mathrm{l})$. Pulmonary hemorrhage, either as isolated capillaritis or as part of the pulmonary renal syndrome, is frequently a life-threatening condition leading to high mortality rates [51]. A retrospective study [57] showed that prompt institution of plasma exchange in addition to immunosuppressive therapy is $100 \%$ life-saving for these patients with pauci-immune glomerulonephritis and diffuse pulmonary hemorrhage when compared to $50 \%$ historical controls. A randomized trial of 137 patients within the European Vasculitis Study Group (EUVAS) with ANCA glomerulonephritis showed a clear benefit of adding plasmapheresis to the standard treatment in patients whose serum creatinine was $>500 \mu \mathrm{mol} / \mathrm{l}$. It was associated with a reduction in the risk for progression to ESRD of $24 \%$ at 12 months. Plasmapheresis was also shown to be a positive predictor of dialysis independence at 1 year in patients with renal failure [58].

Aggressive immunosuppressive therapy is justified by the poor patient and renal survival rates in untreated patients with AAV [41], but toxicity related to therapy is still remarkable. Glucocorticoid therapy is associated with osteoporosis, glucose intolerance and changes in body habitus in the long term, while life-threatening infections occur mainly in the acute phase. The incidence of severe infections is increased from $10 \%$ to $20 \%$ with the addition of cyclophosphamide. The major adverse effects of cyclophosphamide include myelosuppression and hemorrhagic cystitis occurring in $10 \%$ of patients. The bladder cancer rate has been estimated at $5 \%$ in 10 years and at $16 \%$ in 15 years in patients treated with long-term oral cyclophosphamide. Myelodysplasia, lymphoma, skin cancer and gonadal dysfunction are also associated with cyclophosphamide therapy [59]. In order to reduce urotoxicity, certain prophylactic measures have been shown to be helpful, such as sufficient hydration and administration of 2-mercaptoethanesulfonate.

\section{Maintenance Therapy}

Long-standing clinical experience has shown that the disease course varies substantially among patients after achieving remission $[6,8]$. Relapse may occur in $30-$ $50 \%$ of patients achieving remission after the completion of induction therapy. The majority of patients achieve remission, either as a sustained long-term remission, or with one or more relapses occurring over time, while others are prone to persistent, low-grade activity $[8,41]$. More importantly, evaluation of outcomes in patients in whom immunosuppressive therapy was stopped after they attained remission showed a similar rate of relapse compared with patients who remained on treatment for longer periods [8]. In the light of irreversible side effects from therapy and relapse rates being comparable between long-term-treated and untreated responders [8], the decision regarding optimal duration of therapy must be made on an individualized basis. In this regard, maintenance treatment is legitimate for patients who have a high rate of relapse, who have had a relapse already, or those who maintain some disease activity despite full treatment. Recognition of those patients in whom it is safe to discontinue therapy with careful follow-up versus those who require remission maintenance therapy is a clinical conundrum [8]. A practical approach for clinicians is to use predictors of relapse in order to be able to distinguish those patients who are at increased risk of relapse. Predictors of relapse among responders in AAV have been shown to be PR3ANCA seropositivity [20] as well as pulmonary and ear/ nose/throat involvement, each associated with an approximately 2.0 -fold increase in risk for relapse. Importantly, Hogan et al. [8] showed that relapses occurred in $26 \%$ of patients with none of the above risk factors versus $73 \%$ of patients with all of them. 
In order to maintain sustained remission it has been proven that conversion from cyclophosphamide to azathioprine at a dose of $2 \mathrm{mg} / \mathrm{kg}$ of body weight/day is a safe and less toxic choice [9]. Moreover, an open-label randomized controlled trial conducted in 156 patients from 42 centers in 11 European countries showed that mycophenolate mofetil was less effective than azathioprine for maintaining disease remission, while both treatments had similar adverse event rates [60]. Employment of rituximab for the maintenance of remission in patients with AAV was tested in a study of 115 patients with GPA, MPA or renal-limited AAV in complete remission after a cyclophosphamide-glucocorticoid regimen [61]. Patients were randomly assigned to receive either $500 \mathrm{mg}$ of rituximab on days 0 and 14 and at months 6,12 and 18 after study entry or daily azathioprine until month 22 . More patients with AAV had sustained remission at month 28 with rituximab than with azathioprine while the frequencies of severe adverse events were similar in the two groups [61]. Finally, the antimicrobial agent trimethoprim-sulfamethoxazole has been proven to prevent relapse in GPA by reducing the episodes of infections, probably by eliminating Staphylococcus aureus in the upper airways [62].

\section{Therapeutic Options for Persistent, Refractory or Relapsing Disease}

Several agents have been explored as potential alternatives in order to minimize toxicity related to cytotoxic therapy in patients with AAV. Among them, methotrexate combined with corticosteroids showed remission rates of $60-90 \%[63,64]$, but it was associated with an elevated rate of relapse $[53,65]$. Yet, the clinical experience with methotrexate is limited to patients with predominantly extra-renal manifestations of vasculitis and preserved renal function (serum creatinine $<2.5 \mathrm{mg} / \mathrm{dl}$ ). Consequently, patients with any signs of kidney involvement should not receive therapy with methotrexate. Mycophenolate mofetil has been shown to be a safe and therapeutically beneficial alternative for patients with nonlife-threatening, recurrent or resistant AAV in all but 1 of 10 evaluated subjects of a pilot study [66] while considerable relapse rates have been reported by others [67]. Interestingly, adding a moderate dose of cyclosporine to mycophenolate mofetil has been shown to be effective in attaining remission in a small series of patients with relapsing or persistent ANCA-associated glomerulonephritis [68]. In recent years there have been several re-

Pauci-Immune Glomerulonephritis and Vasculitis in Western Countries ports of cases with refractory disease, who were finally managed with rituximab combined with steroids or cyclophosphamide or both and ended up in remarkable improvement [69].

\section{Predictors of Patient and Renal Survival}

Prognosis of AAV is probably the most demanding issue for the clinician and the most concerning for the patients. A prospective study of patients with MPA showed that the relative risk of death was almost nine times greater in patients who presented with pulmonary hemorrhage and four times greater in patients with cytoplasmic versus perinuclear ANCA, while the relative risk of pulmonary hemorrhage was no different by ANCA pattern. The use of cyclophosphamide lowered the risk of death approximately six times when compared with steroid therapy alone [46]. Long-term analysis of patients with GPA who had been treated with prednisone and cyclophosphamide revealed that age $>50$ years at diagnosis, lung or kidney involvement were associated with an almost four-fold increased risk for death [46]. Hogan et al. [46] showed that the strongest predictors of long-term renal survival were entry serum creatinine value, black race and arterial sclerosis on renal biopsy. Although it was clear that the higher the entry serum creatinine the worse the long-term renal prognosis, no specific serum creatinine value could be determined beyond which treatment was not effective, since $50 \%$ of the dialysis-dependent patients at onset recovered renal function, permitting cessation of dialysis [46]. Consequently, prompt institution of immunosuppressive therapy remains the gold standard. The risk for progression to ESRD, after initial response to treatment, in patients with ANCA glomerulonephritis, was a change in the glomerular filtration rate within 4 months of treatment. Specifically, after controlling for baseline creatinine level, type of treatment and ANCA specificity, patients with a glomerular filtration rate decrease of $\geq 8 \mathrm{ml} / \mathrm{min}$ were 5.6 times more likely to progress to ESRD than patients with a stable glomerular filtration rate [8]. Not surprisingly, relapse itself was also shown to increase the likelihood of progression to ESRD by 4.7 times, with the related risk entirely attributable to the recurrence of nephritis [8]. In patients with severe renal dysfunction due to ANCA glomerulonephritis, prognostic indicators of glomerular filtration rate after 12 months were age, percentage of normal glomeruli, tubular atrophy and intraepithelial infiltrates in the renal biopsy [70]. The chances for renal 
recovery were substantially increased by the addition of plasmapheresis [71].

Analysis of clinical, pathological and serological predictors in a large cohort of patients with AAV, recruited by kidney disease, showed that $23 \%$ of the 334 treated patients became treatment-resistant. The majority developed ESRD within a median of 2 months after starting therapy. Female sex, black ethnicity and severity of renal involvement were identified as predictors of treatment resistance. The risk of resistance to immunosuppressive therapy was shown to increase by 1.28 for each serum creatinine elevation of $100 \mu \mathrm{mol} / \mathrm{l}(1.13 \mathrm{mg} / \mathrm{dl})$. Typically, these patients had a relapsing and remitting course not recognized by their primary care provider, leading to advanced glomerular and interstitial scarring at the time of diagnosis. More recently, a study in 155 patients with $\mathrm{AAV}$, who presented with severe renal dysfunction, evaluated the prognostic factors affecting renal and patient outcomes in these patients [72]. Within 4 months after biopsy, treatment response was attained in $51 \%$ of patients, 35\% remained on dialysis, and $14 \%$ died. A competing risk analysis showed that the estimated cumulative incidence rates of ESRD and disease-related mortality were 26 and $17 \%$ at 1 year and 32 and $28 \%$ at 5 years, respectively. Cyclophosphamide therapy and treatment response by month 4 were independently associated with patient and renal survival, adjusting for the percentage of normal glomeruli, histopathological chronicity index score, and baseline clinical characteristics. Only $5 \%$ of patients still dialysis-dependent at 4 months subsequently recovered renal function. Interestingly, among cyclophosphamide-treated patients, the likelihood of treatment response was $>14 \%$ even with highest chronicity index score and estimated glomerular filtration rate $<10 \mathrm{ml} /$ $\min / 1.73 \mathrm{~m}^{2}$. The authors concluded that although low baseline renal function and severe renal scarring are associated with lower treatment response rate, no 'futility' threshold could be identified while continued immunosuppressive therapy beyond 4 months is unlikely to benefit patients who remain dialysis-dependent [72].

\section{Acknowledgements}

We thank our renal pathologist Dr. George Liapis for providing high-quality pictures of ANCA-associated pauci-immune glomerulonephritis.

\section{Disclosure Statement}

The authors declare no conflicts of interest.

\section{References}

1 Jennette JC, Olson JL, Schwartz MM, et al: Primer on the pathologic diagnosis of renal disease; in Jennette JC, Olson JL, Schwartz MM, Silva FG (eds): Heptinstall's Pathology of the Kidney. Philadelphia, Lippincott Williams and Wilkins, 2007, vol 1, pp 97-123.

-2 Jennette JC, Falk RJ, Bacon PA, et al: 2012 revised International Chapel Hill Consensus Conference Nomenclature of Vasculitides. Arthritis Rheum 2013;65:1-11.

-3 Jennette JC, Falk RJ, Andrassy K, et al: Nomenclature of systemic vasculitides. Proposal of an international consensus conference. Arthritis Rheum 1994;37:187-192.

4 Jennette JC: Rapidly progressive crescentic glomerulonephritis. Kidney Int 2003;63: 1164-1177.

5 Jennette JC, Falk RJ: Clinical and pathological classification of ANCA-associated vasculitis: what are the controversies? Clin Exp Immunol 1995;101(suppl 1):18-22.

6 Falk RJ: ANCA-associated renal disease. Kidney Int 1990;38:998-1010.

7 Pettersson EE, Sundelin B, Heigl Z: Incidence and outcome of pauci-immune necrotizing and crescentic glomerulonephritis in adults. Clin Nephrol 1995;43:141-149.
8 Hogan SL, Falk RJ, Chin H, et al: Predictors of relapse and treatment resistance in antineutrophil cytoplasmic antibody-associated small-vessel vasculitis. Ann Intern Med 2005; 143:621-631.

-9 Savage CO, Harper L, Adu D: Primary systemic vasculitis. Lancet 1997;349:553-558.

10 Knight A, Ekbom A, Brandt L, et al: Increasing incidence of Wegener's granulomatosis in Sweden, 1975-2001. J Rheumatol 2006;33: 2060-2063.

11 Watts RA, Mahr A, Mohammad AJ, et al: Classification, epidemiology and clinical subgrouping of antineutrophil cytoplasmic antibody (ANCA)-associated vasculitis. Nephrol Dial Transplant 2015;30(suppl 1):i14-i22.

$\checkmark 12$ Haugeberg G, Bie R, Bendvold A, et al: Primary vasculitis in a Norwegian community hospital: a retrospective study. Clin Rheumatol 1998; 17:364-368.

13 Watts RA, Lane SE, Bentham G, et al: Epidemiology of systemic vasculitis: a ten-year study in the United Kingdom. Arthritis Rheum 2000;43:414-419.
14 Cao Y, Schmitz JL, Yang J, et al: DRB1*15 allele is a risk factor for PR3-ANCA disease in African Americans. J Am Soc Nephrol 2011; 22:1161-1167.

15 Ferrario F, Tadros MT, Napodano P, et al: Critical re-evaluation of 41 cases of 'idiopathic' crescentic glomerulonephritis. Clin Nephrol 1994;41:1-9.

16 Harris AA, Falk RJ, Jennette JC: Crescentic glomerulonephritis with a paucity of glomerular immunoglobulin localization. Am J Kidney Dis 1998;32:179-184.

17 Schnabel A, Holl-Ulrich K, Dalhoff K, et al: Efficacy of transbronchial biopsy in pulmonary vasculitides. Eur Respir J 1997;10:2738-2743.

18 Davies DJ, Moran JE, Niall JF, et al: Segmental necrotising glomerulonephritis with antineutrophil antibody: possible arbovirus aetiology? Br Med J (Clin Res Ed) 1982;285:606.

19 Falk RJ, Jennette JC: Anti-neutrophil cytoplasmic autoantibodies with specificity for myeloperoxidase in patients with systemic vasculitis and idiopathic necrotizing and crescentic glomerulonephritis. N Engl J Med 1988;318:1651-1657. 
20 Lionaki S, Blyth ER, Hogan SL, et al: Classification of antineutrophil cytoplasmic autoantibody vasculitides: the role of antineutrophil cytoplasmic autoantibody specificity for myeloperoxidase or proteinase 3 in disease recognition and prognosis. Arthritis Rheum 2012;64:3452-3462.

21 Lyons PA, Rayner TF, Trivedi S, et al: Genetically distinct subsets within ANCA-associated vasculitis. N Engl J Med 2012;367:214-223.

22 Falk RJ, Terrell RS, Charles LA, Jennette JC: Anti-neutrophil cytoplasmic autoantibodies induce neutrophils to degranulate and produce oxygen radicals in vitro. Proc Natl Acad Sci USA 1990;87:4115-4119.

$\checkmark 23$ Schreiber A, Busjahn A, Luft FC, et al: Membrane expression of proteinase 3 is genetically determined. J Am Soc Nephrol 2003; 14:68-75.

24 Schreiber A, Luft FC, Kettritz R: Membrane proteinase 3 expression and ANCA-induced neutrophil activation. Kidney Int 2004;65: 2172-2183.

25 Charles LA, Caldas ML, Falk RJ, et al: Antibodies against granule proteins activate neutrophils in vitro. J Leukoc Biol 1991;50:539546.

26 Radford DJ, Luu NT, Hewins P, et al: Antineutrophil cytoplasmic antibodies stabilize adhesion and promote migration of flowing neutrophils on endothelial cells. Arthritis Rheum 2001;44:2851-2861.

27 Moon KC, Park SY, Kim HW, et al: Expression of intercellular adhesion molecule- 1 and vascular cell adhesion molecule-1 in human crescentic glomerulonephritis. Histopathology 2002;41:158-165.

28 Xiao H, Heeringa P, Hu P, et al: Antineutrophil cytoplasmic autoantibodies specific for myeloperoxidase cause glomerulonephritis and vasculitis in mice. J Clin Invest 2002;110: 955-963.

-29 Xiao H, Heeringa P, Liu Z, et al: The role of neutrophils in the induction of glomerulonephritis by anti-myeloperoxidase antibodies. Am J Pathol 2005; 167:39-45.

-30 Huugen D, Xiao H, van Esch A, et al: Aggravation of anti-myeloperoxidase antibody-induced glomerulonephritis by bacterial lipopolysaccharide: role of tumor necrosis factoralpha. Am J Pathol 2005;167:47-58.

-31 Jennette JC, Xiao H, Falk RJ: Pathogenesis of vascular inflammation by anti-neutrophil cytoplasmic antibodies. J Am Soc Nephrol 2006 17:1235-1242.

- 32 Xiao H, Schreiber A, Heeringa P, et al: Alternative complement pathway in the pathogenesis of disease mediated by anti-neutrophil cytoplasmic autoantibodies. Am J Pathol 2007;170:52-64.

33 Little MA, Smyth CL, Yadav R, et al: Antineutrophil cytoplasm antibodies directed against myeloperoxidase augment leukocyte-microvascular interactions in vivo. Blood 2005;106: 2050-2058.

Pauci-Immune Glomerulonephritis and Vasculitis in Western Countries
49 Bautz DJ, Lionaki S, Yang JJ, et al: The search for complementary PR3 proteins identified plasminogen as an autoantigen in PR3ANCA disease. J Am Soc Nephrol 2006;17: $85 \mathrm{~A}$. Invest 2013;123:1773-1783.

35 Falk RJ, Hogan S, Carey TS, Jennette JC: Clinical course of anti-neutrophil cytoplasmic autoantibody-associated glomerulonephritis and systemic vasculitis. The Glomerular Disease Collaborative Network. Ann Intern Med 1990;113:656-663.

36 Pendergraft WF, Preston GA, Shah RR, et al: Autoimmunity is triggered by cPR-3(105201), a protein complementary to human autoantigen proteinase-3. Nat Med 2004;10:7279.

37 Woodworth TG, Abuelo JG, Austin HA, et al: Severe glomerulonephritis with late emergence of classic Wegener's granulomatosis. Report of 4 cases and review of the literature. Medicine (Baltimore) 1987;66:181-191.

38 Guillevin L, Lhote F: Classification and management of necrotising vasculitides. Drugs 1997; $53: 805-816$

39 Lhote F, Guillevin L: Polyarteritis nodosa, microscopic polyangiitis, and Churg-Strauss syndrome. Clinical aspects and treatment. Rheum Dis Clin North Am 1995;21:911-947.

40 Fauci AS, Haynes BF, Katz P, Wolff SM: Wegener's granulomatosis: prospective clinical and therapeutic experience with 85 patients for 21 years. Ann Intern Med 1983;98:76-85.

41 Hoffman GS, Kerr GS, Leavitt RY, et al: Wegener granulomatosis: an analysis of 158 patients. Ann Intern Med 1992;116:488-498.

42 Leavitt RY, Fauci AS, Bloch DA, et al: The American College of Rheumatology 1990 criteria for the classification of Wegener's granulomatosis. Arthritis Rheum 1990;33:11011107.

43 Nachman PH, Reisner HM, Yang JJ, et al: Shared idiotypy among patients with myeloperoxidase-anti-neutrophil cytoplasmic autoantibody associated glomerulonephritis and vasculitis. Lab Invest 1996;74:519-527.

44 Savage CO, Winearls CG, Evans DJ, et al: Microscopic polyarteritis: presentation, pathology and prognosis. Q J Med 1985;56:467-483.

45 Daha MR, Falk RJ: Anti-myeloperoxidase antibodies and clinical associations. Neth J Med 1990;36:152-153.

46 Hogan SL, Nachman PH, Wilkman AS, et al: Prognostic markers in patients with antineutrophil cytoplasmic autoantibody-associated microscopic polyangiitis and glomerulonephritis. J Am Soc Nephrol 1996;7:23-32.

47 Merkel PA, Lo GH, Holbrook JT, et al: Brief communication: high incidence of venous thrombotic events among patients with Wegener granulomatosis: the Wegener's Clinical Occurrence of Thrombosis (WeCLOT) Study. Ann Intern Med 2005;142:620-626.

48 Weidner S, Hafezi-Rachti S, Rupprecht HD: Thromboembolic events as a complication of antineutrophil cytoplasmic antibody-associated vasculitis. Arthritis Rheum 2006;55:146149.
50 Falk RJ, Hoffman GS: Controversies in small vessel vasculitis - comparing the rheumatology and nephrology views. Curr Opin Rheumatol 2007;19:1-9.

-51 Nachman PH, Hogan SL, Jennette JC, et al Treatment response and relapse in antineutrophil cytoplasmic autoantibody-associated microscopic polyangiitis and glomerulonephritis. J Am Soc Nephrol 1996;7:33-39.

52 Novack SN, Pearson CM: Cyclophosphamide therapy in Wegener's granulomatosis. N Engl J Med 1971;284:938-942.

53 de Groot K, Jayne DR, Tesar V, Savage CO: European, multicenter randomised controlled trial of daily oral versus pulse cyclophosphamide for induction of remission in ANCA-associated systemic vasculitis. J Am Soc Nephrol 2005;16:7A

54 Harper L, Morgan MD, Walsh M, et al; EUVAS investigators: Pulse versus daily oral cyclophosphamide for induction of remission in ANCA-associated vasculitis: long-term follow-up. Ann Rheum Dis 2012;71:955-960.

55 Jones RB, Tervaert JW, Hauser T, et al; European Vasculitis Study Group: Rituximab versus cyclophosphamide in ANCA-associated renal vasculitis. N Engl J Med 2010;363:211220

56 Stone JH, Merkel PA, Spiera R, et al; RAVEITN Research Group: Rituximab versus cyclophosphamide for ANCA-associated vasculitis. N Engl J Med 2010;363:221-232.

57 Klemmer PJ, Chalermskulrat W, Reif MS, et al: Plasmapheresis therapy for diffuse alveolar hemorrhage in patients with small-vessel vasculitis. Am J Kidney Dis 2003;42:1149-1153.

58 de Lind van Wijngaarden RA, Hauer HA, Wolterbeek R, et al: Clinical and histologic determinants of renal outcome in ANCA-associated vasculitis: a prospective analysis of 100 patients with severe renal involvement. J Am Soc Nephrol 2006;17:2264-2274.

59 Beimler JH, Andrassy K: Cyclophosphamide treatment in systemic necrotizing vasculitis and lupus nephritis. How long? How much? Pediatr Nephrol 2004;19:949-955.

60 Hiemstra TF, Walsh M, Mahr A, et al; European Vasculitis Study Group (EUVAS): Mycophenolate mofetil vs azathioprine for remission maintenance in antineutrophil cytoplasmic antibody-associated vasculitis: a randomized controlled trial. JAMA 2010;304: 2381-2388.

61 Guillevin L, Pagnoux C, Karras A, et al; French Vasculitis Study Group: Rituximab versus azathioprine for maintenance in $\mathrm{AN}$ CA-associated vasculitis. N Engl J Med 2014; 371:1771-1780. 
62 Stegeman CA, Tervaert JW, De Jong PE, et al: Trimethoprim-sulfamethoxazole (co-trimoxazole) for the prevention of relapses of Wegener's granulomatosis. Dutch Co-Trimoxazole Wegener Study Group. N Engl J Med 1996;335:16-20.

-63 Langford CA, Talar-Williams C, Barron KS, et al: A staged approach to the treatment of Wegener's granulomatosis: induction of remission with glucocorticoids and daily cyclophosphamide switching to methotrexate for remission maintenance. Arthritis Rheum 1999;42:2666-2673.

64 Langford CA, Talar W, Sneller MC: Use of methotrexate and glucocorticoids in the treatment of Wegener's granulomatosis. Longterm renal outcome in patients with glomerulonephritis. Arthritis Rheum 2000;43:18361840.
65 Furst DE: Practical clinical pharmacology and drug interactions of low-dose methotrexate therapy in rheumatoid arthritis. Br J Rheumatol 1995;34(suppl 2):20-25.

66 Joy MS, Hogan SL, Jennette JC, et al: A pilot study using mycophenolate mofetil in relapsing or resistant ANCA small vessel vasculitis. Nephrol Dial Transplant 2005;20:2725-2732.

67 Koukoulaki M, Jayne DR: Mycophenolate mofetil in anti-neutrophil cytoplasm antibodies-associated systemic vasculitis. Nephron Clin Pract 2006;102:c100-c107.

68 Cooper JD, Hogan SL, Chin H, et al: Mycophenolate mofetil plus cyclosporine therapy for active ANCA vasculitis. J Am Soc Nephrol 2006; 17:547A.
69 McGregor JG, Hogan SL, Kotzen ES, et al: Rituximab as an immunosuppressant in antineutrophil cytoplasmic antibody-associated vasculitis. Nephrol Dial Transplant 2015;30 (suppl 1):i123-i131.

70 Jayne D, Rasmussen N, Andrassy K, et al: A randomized trial of maintenance therapy for vasculitis associated with antineutrophil cytoplasmic autoantibodies. N Engl J Med 2003; 349:36-44.

71 Reinhold-Keller E, Beuge N, Latza U, et al: An interdisciplinary approach to the care of patients with Wegener's granulomatosis: longterm outcome in 155 patients. Arthritis Rheum 2000;43:1021-1032.

72 Lee T, Gasim A, Derebail VK, et al: Predictors of treatment outcomes in ANCA-associated vasculitis with severe kidney failure. Clin J Am Soc Nephrol 2014;9:905-913. 